\title{
COMPUTER SIMULATIONS OF FREEZING AND SUPERCOOLED LIQUIDS
}

\author{
D. Frenkel and J. P. McTague \\ Department of Chemistry, University of California, Los Angeles, California \\ 90024
}

\section{INTRODUCTION}

The solid-liquid transition is one of the most common phase changes occurring in nature, yet our theoretical understanding of it is quite fragmented. Although there exist theories of freezing and of melting, these theories are often phrased in very different languages. In order to understand the thermodynamics and kinetics of the solid-liquid transition it is essential to have a good theoretical picture of the microscopic structure and dynamics of both the solid and the liquid phase, along the melting curve. The theoretical description of anharmonic, defect-rich solids and, in particular, of dense fluids is a formidable many-body problem. It is in tackling this problem that simulation techniques have proven to be an invaluable tool. In discussing the role of (computer) simulations in the study of the solid liquid transition, it is important to distinguish between the thermodynamics and the kinetics of the phase transition. Studies of the thermodynamics of the solid-liquid transition address themselves to the following question: Given the intermolecular forces for a particular system, what is the location of the melting curve and how do the thermodynamic properties of the system (e.g. density, entropy etc) change on melting? In contrast, studies of the kinetics of the solid-liquid transition focus on the question: By what microscopic mechanism does a system melt or freeze? Of course, as many fluids can be cooled well below their normal freezing point, related questions are: What factors favor supercooling and what is the nature of the glass transition? In general, the answers to these questions may be expected to depend on the dimensionality of the system and the nature of the intermolecular forces. 
The outline of the present review is as follows. First we briefly discuss the simulation techniques that are used to study the above mentioned aspects of the solid-liquid transition. Next we present a necessarily incomplete survey of the relevant simulation results. In this survey we limit ourselves to single component systems and largely, though not exclusively, to molecules interacting through central forces. The material is organized in three sometimes overlapping categories: thermodynamics, kinetics, and the properties of amorphous solids. Although a survey of the theoretical framework (or frameworks) is clearly outside the scope of this review, we attempt to assess the relevance of simulations on finite systems as a means to test theoretical predictions on infinite systems. Not included in this survey are the freezing and melting properties of mixtures, liquid-crystals, polymeric substances, and quantum systems.

\section{SIMULATION METHODS}

\section{Mechanical Models}

The earliest simulations of the microscopic structure of dense fluids were mechanical rather than numerical in nature. Bernal, who pioneered the structural analysis of assemblies of randomly packed spheres, quotes in his 1962 Bakerian Lecture (1) the work of the Reverend Stephen Hales who, in 1727, studied the swelling properties of peas. The Reverend observed that peas, when made to swell in a heavy iron pot with a weighted lid, tended to fill up the remaining space, "being thereby formed into pretty regular Dodecahedrons." In the early 1940s, Rice determined the density of an assembly of glass beads randomly thrown into a container and then shaken down (2). He found that the density of this assembly of spheres was about $15 \%$ lower than that of the closepacked crystalline phase [earlier experiments by Westman \& Hugill (3) had yielded a value some $21 \%$ below the crystal density]. On basis of this observation Rice predicted that, at sufficiently high pressures, the hard-sphere solid must be more stable than the fluid phase. More recently, mechanical models of hard-sphere fluids were investigated in great detail by Bernal $(1,4-7)$ and Scott et al (8-11), and we discuss some of their findings in the section on amorphous solids. At this stage we only want to sketch the method and point out some of its advantages and limitations.

Mechanical models can be used to study some of the structural properties of hard-core fluids. As an example, let us consider an assembly of ball bearings. This system has been extensively used to simulate a dense hard-sphere fluid. In a mechanical simulation a large 
number of spheres [typically $\Theta\left(10^{4}\right)$ ] are poured into a container with irregularly shaped walls (to prevent "nucleation"). On "shaking and tapping," (8) the density of the assembly quickly levels off at a maximum (the density of "random close packing"). Of course, as the system is finite, boundary effects may be important and a careful extrapolation to infinite system size is important (8). Properties that can be conveniently studied with a mechanical model are the density of random close packing $(12,13)$ and the local coordination geometry of particles. Extracting further structural information (e.g. the radial distribution function) from a mechanical model is exceedingly tedious. Moreover, mechanical models can only be used to simulate a system at the density of random close packing (assuming that this state is sufficiently well defined). The "correct" preparation of a random close structure is not well understood; other preparation methods lead to lower final densities, although under shearing motion both 3-D (11) and 2-D (14) random close packed structures were found to "crystallize."

\section{Computer Simulations}

Almost from the day that they were first introduced, computer simulation techniques have been used to study the solid-liquid transition and related phenomena. In 1953 Metropolis et al (15) reported the first Monte Carlo (MC) simulation on a system of 224 hard disks. The first Molecular Dynamics (MD) calculations (on systems of up to 108 hard spheres) were reported by Alder \& Wainwright in 1957 (16). Excellent review articles exist on both the MC (17-19) and the MD (20-22) method, to which we refer the reader for information on the practical aspects of these simulation techniques. Here we only describe the basics of the MC and MD methods and mention certain practical limitations of these simulation techniques that may have important consequences for the study of phase transitions (see also 23, chapter 10).

As the MC and MD methods are closely related, we briefly sketch the MD method (because it is conceptually simpler) and, after that, indicate in what respects the MC method is different.

A Molecular Dynamics simulation amounts to nothing more than a numerical integration of the classical equations of motion for an assembly of $N$ particles. To start a simulation one has to specify: $(a)$ the positions and momenta (and, for molecules, orientations, and angular momenta) of all particles and $(b)$ the intermolecular force laws (in general assumed to be pairwise additive). The force on each particle can then be evaluated by summing the contributions due to all other particles within a distance $r_{c}$ from that particle, where $r_{c}$ is a cutoff distance appropriate to the range of the force. Given the force on each 
particle the equations of motion can be integrated for a small, but finite time increment. The new positions are then used to evaluate the new forces and the cycle is repeated. (Note: MD calculations on particles with steep repulsive cores follow a somewhat different procedure. In the latter case the equations of motion are integrated from one collision to the next.) The most important limitation of the MD (and MC) method is the fact that only relatively small systems $\left(10^{2}\right.$ to $10^{4}$ particles) can be handled with the present generation of computers. As a consequence, boundary effects may be very important (the fraction of atoms at the surface is proportional to $N^{-1 / D}$, where $D$ is the dimensionality of the system). For this reason periodic boundary conditions are commonly used in the simulation of bulk fluids (solids). The effect of periodic boundary conditions is to embed the system to be studied in an infinite array of identical replica systems. The most common periodic boundary condition is the cubical (sometimes rectangular) boundary condition; $N$ particles are initially placed in a cube (length $L$ ). This cube is the unit cell of a simple cubic lattice of replica systems. Every time a particle moves out of the original cube (say at $x=+L / 2$ ) its periodic image will move into cube (at $x=-L / 2$ ). Although periodic boundary conditions effectively eliminate surface effects, they do introduce some problems of their own. For instance, a periodic fluid is no longer isotropic but has the symmetry of the boundary conditions (e.g. cubic). Moreover, crystal lattices that are commensurate with the boundary conditions are clearly favored over all others. When studying the solid-liquid transition in a periodic system one should attempt to assess the effect of the boundary conditions. Mandell (24a) has studied the effect of periodic boundary conditions on the properties of a fluid of 108 Lennard-Jones molecules. $\mathrm{He}$ found that for such a small system the periodic boundary effects are by no means negligible, in particular at low temperatures.

Another effect of periodic boundary conditions is the suppression of all fluctuations with wavelengths larger than the boxlength $L$ [or, to be more precise, only fluctuations with wavevector $\mathrm{k}=(2 \pi / L)\left(n_{x}, n_{y}, n_{z}\right)$ are allowed]. It should be stressed that periodic boundary conditions only eliminate surface effects in a one-phase system. If two phases coexist, the fraction of particles in the interface region is once again of order $N^{-1 / D}$. Consequently, a system that may be large enough to study the properties of a single phase may be much too small to observe two-phase coexistence. An alternative to periodic boundary conditions is to constrain the system to a closed (hyper) surface. This latter method has been used by Hansen, Levesque \& Weis (24b) who studied a two-dimensional one-component plasma, constrained to move on the surface of a sphere. In an MD calculation the total energy $E$, the volume $V$, and the number of particles $N$ are held constant. If the 
system is ergodic, taking the time average of a dynamical property will be equivalent to taking the microcanonical ensemble average. Two questions arise: 1 . Is the system under consideration indeed ergodic? 2. If so, how long should one time-average to obtain a result within specified error bounds? In general there is no satisfactory answer to either of these questions. The first question, although interesting in principle, is less relevant for MD calculations than the second. At high densities and low temperatures finite periodic systems with a sufficiently repulsive core will, most likely, become nonergodic (i.e. the available phase space consists of a number of isolated islands). But what really counts is whether the system can adequately sample the available phase on the time scale of a typical MD simulation. If the longest relaxation times in the system become of the order of the duration of an MD run, the computed time average will start to deviate appreciably from the microcanonical ensemble average. Very long relaxation times may be expected near a critical point or close to the glass transition. Under such circumstances one may also expect a pronounced system-size dependence of the observed properties, because the longest relaxation time is of ten strongly size dependent.

Another manifestation of nonergodicity on the time scale of an MD experiment is metastability. The distinction between metastability and the examples given above is that there is a large gap between all the relaxation times of the metastable phase and the characteristic time it takes to nucleate the stable phase. The properties of metastable phase (e.g. a supercooled liquid) can be studied by MD simulation in the same way as those of a stable phase. However, when the gap between relaxation and nucleation rates becomes smaller it is no longer meaningful to speak of a metastable phase.

Monte Carlo simulations are used to study the static properties of systems at constant $N, V$, and $T$ (the method can easily be adapted to study constant $N, P, T$ or $\mu, V, T$ systems). In an MC simulation the system performs a biased random walk through phase space. In the basic MC step one particle in the system is given a small, random displacement. The potential energy of the resulting configuration is then compared with the original potential energy. If the move lowers the potential energy, the move will be accepted; if it increases the potential energy by an amount $\Delta U$ the move is rejected unless $\exp (-\Delta U / k T)>R$ (where $R$ is a random number between 0 and 1). Irrespective of whether or not the move was accepted, the properties of the resulting configuration are included in the averaging.

The systems studied by MC calculations are typically of the same size as those studied by Molecular Dynamics and periodic boundary conditions are usually imposed. The important difference between the MC 
and MD methods is that the molecular movements in an MC simulation are unrelated to the natural time evolution of the system. Consequently, no dynamical information can be derived from MC simulations. An infinite MC simulation will yield average values for the energy, pressure and other static properties that are identical to the canonical ensemble averages (for a constant $N, V, T$ simulation) $(15,18)$. As in the MD case it may happen that the available phase space is inadequately sampled in a finite MC run. In general, such problems have been observed under the same conditions that lead to nonergodic behavior in MD simulations. Yet it is not obvious that this should always be the case because in MC runs "structural relaxation" and "nucleation" are the result of a succession of nonphysical moves. Consequently, structural rearrangements that are favored by the MC method may be impossible to realize in an MD simulation, and vice versa. Apart from the MD and MC methods a number of computation techniques have been developed to study the properties of dense random-packed structures. Such computer modeling techniques have recently been reviewed in a paper by Hoare (25), to which we refer the interested reader.

\section{THERMODYNAMICS OF MELTING}

Locating the melting curve of a model system in an MC or MD simulation is not a trivial matter. When Metropolis et al first studied the equation of state of 224 hard disks (15), they passed through what is now believed to be the melting density of this system without finding evidence for a phase transition. In their description of the first Monte Carlo simulations of a system of 256 hard spheres, Rosenbluth \& Rosenbluth reported that the equation of state shows no sign of a phase transition (26). But at the same time they observed that the radial distribution function changes from "solid-like" to "liquid-like"when the volume of the system is expanded to about 1.5 times the regular close packed volume, $V_{0}$. [In fact, the best estimates of the volume of a hard-sphere fluid at coexistence yield $V / V_{0}=1.50$ (27).] Evidence for the existence of discontinuous solid-fluid transition came from the first Molecular Dynamics simulation of hard-sphere system by Alder \& Wainwright (16) and an accompanying paper by Wood \& Jacobson (28) who performed extensive Monte Carlo calculations on the same system. It was observed in both these calculations that for $1.5 \leqslant V / V_{0} \leqslant 1.6$ the hard-sphere system can be in either a "low-pressure" state or a "highpressure" state. The low-pressure and high-pressure points define separate branches of the equation of state, and jumps between the two branches occur very infrequently and only in a limited density range. 
Both papers point out that on the high-pressure branch molecular diffusion is relatively free, whereas in the low-pressure system the spheres are confined by their neighbors. Yet, the words "solid" and "fluid" are never used in either paper.

These early simulations illustrate some of the problems associated with the determination of the melting point. In the small systems studied in References $(16,28)(N=32$ and $N=108)$, two-phase coexistence is apparently impossible because of the large amount of free energy needed to form an interface. Although calculations of this sort can be used to estimate a lower limit to the density of the stable solid phase, they provide no direct information on the densities of the coexisting liquid and solid phases. To observe coexisting phases one has to study fairly large systems. Alder \& Wainwright (29) observed twophase behavior in a system of 870 hard disks. In three dimensions, one has to go to even larger systems to observe coexistence. For instance, solid-fluid coexistence has recently been observed by Cape \& Woodcock (30) in a system of 1920 soft-spheres $\left[\phi(r)=\varepsilon(\sigma / r)^{12}\right]$. Ladd \& Woodcock $(31,32)$ even reported three-phase coexistence in a system of 1500 Lennard-Jones (LJ) atoms. Although direct observation of twophase coexistence may seem a straightforward method to study the properties of the coexisting phases, there are some disadvantages attached to it. In a macroscopic system, the $P$ vs $\rho$ isotherm is flat in the coexistence region. For the system sizes studied in MD and MC simulations, $P$ is not constant in the coexistence region, but exhibits a "van der Waals-like" loop (29). This loop is a consequence of the fact that the free energy needed to form interfaces is non-negligible in a small system [the relation between the shape of the loop and the solid-fluid surface tension has been discussed in some detail by Mayer \& Wood (33)]. Alder \& Wainwright determined the densities of the coexisting phases from an equal area construction. To get acceptable statistics on the pressure in the two-phase region, exceedingly long runs (up to $10^{7}$ collisions/run) are required because the dynamics in the two-phase region is quite sluggish. Woodcock and co-workers determined the density of the coexisting phases directly by studying parts of the solid and liquid phases not too close to the interface. The latter method, although more economical because only one state is studied, requires careful preparation of a stable, equilibrated two-phase system as the differences between an insufficiently equilibrated sample (31) and a well-equilibrated one (32) are quite substantial. Woodcock et al give no estimate of the effect of the solid-fluid interface on the total pressure. The Alder-Wainwright method, although straightforward, is prohibitively expensive. 
For these reasons many of the computer studies of melting have concentrated on methods to determine the solid-fluid coexistence curve from properties of the homogeneous phases only. Here we should distinguish between rigorous and approximate melting criteria. The rigorous thermodynamic criterion for solid-fluid coexistence is that the chemical potential of the two phases at equal temperature and pressure be the same. The well-known double tangent construction to the free energies of the coexisting phases is the graphical expression of this condition. Unfortunately neither the MC nor the MD method directly determine the free energy [to be precise, the $(\mu, V, T)$ Monte Carlo method does, but is impractical at the relevant densities (19)]. To determine the free energy of the solid and fluid phase near the melting curve one has to start from a reference state for which the free energy is known exactly (e.g. the ideal gas) and then compute the difference between the free energy of the dense fluid (solid) and the reference state (for instance, by integrating $(\partial A / \partial V)_{T}=-P$ along an isotherm). Two, rather similar, methods have been developed to calculate the free energy of both solid and fluid phases. One method originally introduced by Hoover, Gray \& Johnson (34) to determine the melting properties of systems with inverse power interactions $\left[\phi(r)=\varepsilon(\sigma / r)^{n}\right.$, with $n=4,6$, and 9] uses the ideal gas as the reference state for the fluid and the dense, harmonic solid as the reference state for the solid. Of course, determining the free energy of the (classical) harmonic solid is nontrivial because it involves finding the complete phonon density of states. An alternative method that can also be applied in cases where the dense solid is not harmonic (e.g. a hard-sphere system) was developed somewhat earlier by Hoover \& Ree (35). In this method the reference state for the fluid is also the ideal gas. But the free energy of the solid is determined by constructing a reversible path from the dilute gas to the dense solid. This is accomplished using an artificial single-occupancy solid. In such a solid the entire volume is divided up into Wigner-Seitz cells corresponding to the lattice type of the solid. In the MC or MD calculation each particle is constrained to stay within its cell. In the dense solid these constraints have no noticeable effect on the thermodynamic properties because the particles are localized by their neighbors and collisions with cell walls are very unlikely. This single-occupancy solid is slowly expanded from solid densities to dilute gas densities. Due to the presence of the cell walls, melting, in the conventional sense of the word, does not take place. Hoover \& Ree noted that the equation of state of the single-occupancy solid shows a cusp at the density where the localization of the molecules becomes dominated by the cell walls [to be precise, this cusp is well defined in 3-D, but somewhat fuzzy in 2-D 
$(27,36)]$. The advantage of the Hoover \& Ree method is that it is simple and applicable to any system that forms an ordered solid at sufficiently high densities. Hoover et al (37) compared the lattice dynamics method and the single-occupancy cell technique for a system of "soft-spheres" $\left[\phi(r)=\varepsilon(\sigma / r)^{12}\right]$ and found good agreement. Nevertheless caution is required when using the single-occupancy model for the following reasons:

1. It is not obvious that the equation of state of the single-occupancy solid is necessarily free of discontinuities [see, for instance, the MC calculations by Ogura et al (38)].

2. The single-occupancy model is not a valid description of the solid phase if lattice defects contribute appreciably to the thermodynamic properties (this may happen close to the melting transition).

3. Because of the lack of long-range translational order in infinite 2-D solids at finite temperatures (39), the single-occupancy model may be less appropriate in two dimensions.

An important problem is the $N$ dependence of the location of the melting curve. Hoover \& Alder (40) observed that the melting pressure in a system of 870 hard disks was some $10 \%$ higher than in a system of 72 disks [see also the $(N, P, T)$ MC calculations by Wood (41; see also 17)]. Hoover \& Alder argued that the main $N$ dependence of the transition is due to the $N$ dependence of the communal entropy of the fluid phase (even though the communal entropy is not the most important contribution to the entropy of fusion). The leading $N$ dependent term in the communal entropy is $-1 / 2 \ln (2 \pi N) / N$. This expression predicts an $N$ dependence of the hard-disk melting point that agrees closely with the MD data of Reference (40). This $\ln N / N$ dependence is probably quite general because the free energy of a harmonic solid also has a leading $N$ dependence of order $\ln N / N$ (37). Finally it should be emphasized that methods like the ones mentioned above are dangerous to use if it is not obvious that the melting transition is first order. As the method sets out to determine the separate free energy branches for the solid and fluid phases, slight inaccuracies in the results may lead one to interpret a higher order transition as being weakly first order. This problem seems to be particularly serious for the 2-D one-component plasma (42).

The above discussion clearly indicates that all "rigorous" methods to determine melting curves are quite time consuming, requiring either lengthy runs on large, two-phase systems or runs on a large number of thermodynamic states of the (artificial) one-phase systems. For this 
reason there have been a number of attempts to correlate melting to a directly observable property of either the solid or the fluid phase.

The best known criterion of this nature is Lindemann's melting law (43). This law states that along the melting curve the rms displacement of a molecule from its lattice site is a fixed fraction of the lattice spacing. This criterion has been tested in a number of computer simulations $(34,36,42,44-46)$. In general it is found that, for a given substance, the fraction $f=\left\langle(\Delta r)^{2} / d^{2}\right\rangle^{1 / 2}$ is fairly constant along the melting curve (for inverse power potentials, rigorously constant), but for different model systems $f$ may be quite different [e.g. $f \approx 0.136$ for hard spheres (45) and $f \approx 0.176$ for the classical one-component plasma (46)]. In two dimensions the Lindeman criterion is, strictly speaking, not applicable because $\left\langle(\Delta r)^{2}\right\rangle$ is expected to diverge as $\ln N$. This $N$ dependence has indeed been verified $(42,45)$ but, for a given system size, $f$ is found to be reasonably constant (36) and, suprisingly, even for rather different systems [hard disk (45), $f \approx 0.17 ; 2-\mathrm{D} \mathrm{LJ}(36), f \approx 0.15$; 2-D classical one-component plasma (42), $f=0.176$; all evaluated for $N=256]$. Another melting criterion that focuses on the properties of the solid phase at melting has been formulated by Ross (47). Ross' melting rule states that, at melting, the excess free energy $A_{e x}$ is constant. $A_{e x}$ is defined as

$$
\frac{A_{e x}}{N k T}=\frac{A}{N k T}-\ln \left(\frac{N}{V}\right)+1-\frac{V_{o}}{N k T}
$$

where $A$ is the total free energy of the solid and $V_{0}$ is the potential energy of the solid if all molecules are localized at the lattice sites. Ross' criterion presupposes knowledge of the free energy of the solid as a function of $\rho$ and $T$ and is therefore not exactly a simple rule of thumb. Where it has been tested it appears to be somewhat superior to Lindemann's rule. Hoover, Gray \& Johnson (34) find that for systems of inverse power potentials $(n=4,6,9,12$ and $\infty), A_{e x}$ is very close to $6 N k T$ (the extreme value is $6.3 N k T$ for $n=4$ ). In contrast to Lindemann's rule, Ross' criterion can be used in 2-D; for hard disks $A_{e x}=3.9 N k T$ (27), for two-dimensional Lennard-Jones molecules $A_{e x} \approx 3.7 N k T$ (36).

A freezing criterion based on the properties of the dense fluid has been formulated by Hansen \& Verlet (48) and investigated in some detail by Hansen \& Schiff (49). The rule states that, along the melting curve, the value of the first maximum of the structure factor is constant and approximately equal to the first maximum of the structure factor of a hard-sphere fluid at the freezing density. This rule is based on the same observation that provides the starting point for the successful perturbation theories of dense simple liquids $(50,51)$ and the Longuet- 
Higgins-Widom theory of freezing (52), namely that the structure of the dense fluid is dominated by the steep repulsive interactions between molecules. In fact, as has first been shown by Verlet (53), an appropriate choice of the equivalent hard-sphere radius can make the structure factor of a hard-sphere fluid almost indistinguishable from that of a dense Lennard-Jones fluid, in particular close to its first maximum. At freezing, the first peak in the structure factor of a hard-sphere fluid is $S\left(k_{o}\right)=2.85$ (49). Hansen \& Verlet (48) observed that, for a LennardJones system, the criterion $S\left(k_{o}\right)=2.85$ predicts the correct shape of the melting curve over a wide range of pressures. Later work by Hansen $\&$ Schiff indicates that for widely different inverse power potentials $(\phi=$ $\left.\varepsilon(\sigma / r)^{n}, n=1,4,6,9,12, \infty\right) S\left(k_{o}\right)$ at melting varies between $3.05(n=12)$ and $2.57(n=1)$. The Hansen-Verlet melting criterion has not been adequately tested in 2-D. Gann et al (42) have studied the structure factor of the classical 2-D one-component phases. They could not obtain a good estimate of $S\left(k_{o}\right)$ at melting but suggest that it is certainly larger than the 3-D hard-sphere value of 2.85 . (Curiously enough, the 2-D hard-disk value has, to our knowledge, not been published.) Hockney \& Brown (54) obtain very different estimates for the structure factor of the 2-D one-component plasma (OCP) (and, in fact, for all other properties) but their plots of $S(k)$ suggest that a nonconventional definition of the structure factor was used. Systematic tests of the 2-D equivalent of the Hansen-Verlet rule would be interesting because liquid state perturbation theories are not nearly as successful in 2-D as they are in 3-D (55). Raveche, Mountain \& Streett (56) observed that for an $\mathrm{LJ}$ fluid near freezing, the ratio

$$
R=g\left(r_{\min }\right) / g\left(r_{\max }\right) \approx 0.2
$$

where $r_{\min }$ is the position of the first (nonzero) minimum of the pair distribution function and $r_{\max }$ the position of its first maximum. Raveche et al quote experimental evidence indicating that for some real simple liquids this criterion is valid to within $\pm 10 \%$. The data of Hansen \& Schiff for inverse power potentials indicate that $R$ varies from 0.10 for hard spheres $(n=\infty)$ to $\mathrm{R}=0.26$ for the classical OCP $(n=1)$.

Two heuristic melting criteria have been proposed specifically for the purpose of quickly and economically locating melting points in machine calculations. The first criterion, due to Ross \& Alder (44), states that the highest density at which an over-expanded solid can melt during a typical Monte Carlo simulation coincides with the fluid density at coexistence. A similar rule has been formulated by Streett, Raveche \& Mountain (57). This rule also attributes special significance to the instability point of the overexpanded solid. It suggests that the fluid and 
solid branches of $P$ vs $V$ may be joined by drawing a vertical line at the density of instability. The densities of the coexisting phases are than determined by an equal area construction on the resulting "van der Waals loop." For an LJ system the melting density thus obtained is some $4 \%$ lower than the value derived by Hansen \& Verlet from a double-tangent construction on the free energy; the melting pressures even differ by some $30 \%$. The method of Streett et al has been criticized by Hansen \& Pollock (58). If melting is not a first-order transition none of the above melting criteria are applicable. In such cases it is advantageous to monitor directly the mechanical and transport properties of the two phases because, in contrast to the first-order case, metastability cannot occur. It should be noted, however, that close to a higher order transition the structural relaxation becomes very sluggish and apparent hysteresis may result if the transition region is traversed too fast. Of course, one does not always know whether a transition will be higher order or weakly first-order but, as long as hysteresis effects are small, monitoring mechanical and transport properties is a sensitive way of detecting phase transition in either case. Such properties are for instance the shear modulus of the system (59), the shear viscosity (60), the self-diffusion constant (59), and the order-parameters indicative of long-range translational and orientational order (60).

\section{SPECIFIC EXAMPLES}

For a number of simple model systems the melting curve has been determined using one or more of the techniques described above. The systems that have been studied most extensively are those with inverse power potential interactions: $v(r)=\varepsilon(\sigma / r)^{n}$. To this class belong both the hard-sphere (disk) system $(n=\infty)$ and the classical one-component plasma $(n=1)$. Due to the scaling properties of the inverse power potentials, the equation of state of these systems is a function of one parameter only: $(\varepsilon / k T)^{D / n} \sigma^{D} p$. It suffices therefore to determine one melting point, because the coexistence curve follows the scaling relation $\rho_{F}=$ constant $\left(k T_{F} / \varepsilon \sigma^{n}\right)^{D / n}$.

If several solid phases with different crystal structures are possible, coexistence between the fluid and the most stable crystal phase defines the melting curve. Hoover, Young \& Grover (61) have investigated the relative stability of $\mathrm{FCC}$ and $\mathrm{BCC}$ phases for inverse power potentials. They find that for $n \gg 6, \mathrm{BCC}$ is the more stable solid at high temperatures, whereas for $n>7$ the BCC phase is unstable. The relative stability of the different close-packed phases (e. g. FCC, HCP) at high temperatures has not been investigated for most inverse power potentials with 
$n>7$. In general it is assumed that FCC is the stable solid at melting. For hard spheres $(n=\infty)$ there is some evidence that, at close-packing, the entropy of the FCC solid is slightly higher than that of the HCP solid (45). This difference is, however, extremely small $\left(S_{F C C}-S_{H C P} \approx\right.$ $0.002 N k$ ), and for system sizes that are typically studied in machine calculations $\left[N=\theta\left(10^{3}\right)\right]$ the entropy of hybrid FCC-HCP phase would be higher than that of the pure phases.

The coexistence properties of a number of inverse power potential systems have been collected in Table 1. Additional information on the phase coexistence properties of inverse power potential spheres can be found in the following References: $(16,17,20,27,28,35,62-64)$ for hard spheres; $(30,34,37,38,49,65,66)$ for inverse power potentials; $(46,67,68)$ for 3-D one-component plasma; $(17,27,29,35,41)$ for 2-D hard-disks; $(42,54,59)$ for 2 -D one-component plasma; and (69) for 2-D inverse power potentials.

The phase diagram of the 3-D Lennard-Jones system has been studied extensively by several authors $(31,32,48,57,65)$. Hansen \& Verlet performed single-occupancy cell calculations for four different isotherms (48), while Ladd \& Woodcock studied three-phase coexistence in a large system $(N=1500)(31,32)$. Single-occupancy cell MD calculations on a 2-D Lennard-Jones fluid have been performed by Toxvaerd (36) for 5 different temperatures. In the temperatures range studied by Toxvaerd the melting transition appears to be first-order. Earlier MC

Table 1 Coexistence properties of a number of inverse power potential systems

\begin{tabular}{|c|c|c|c|c|}
\hline \multirow[t]{2}{*}{ 3-D } & \multirow[b]{2}{*}{$\left(\rho_{s} / \rho_{o}\right)\left(\varepsilon / k T_{m}\right)^{3 / n}$} & \multirow[b]{2}{*}{$\left(\rho_{F} / \rho_{o}\right)\left(\varepsilon / k T_{m}\right)^{3 / n}$} & \multirow[b]{2}{*}{$P / \rho_{o} k T_{m}$} & \multirow[b]{2}{*}{$\left(\rho_{o} \sigma^{3}=\sqrt{2}\right)$} \\
\hline & & & & \\
\hline$n=\infty$ & 0.736 & 0.667 & 8.27 & Ref. (27) \\
\hline$n=12$ & 0.844 & 0.813 & 16 & Ref. $(34,37,65)$ \\
\hline$n=9$ & 0.971 & 0.943 & 22 & Ref. (34) \\
\hline$n=6$ & 0.156 & 1.54 & 61 & Ref. (34) \\
\hline$n=4$ & 3.94 & 3.92 & 426 & Ref. (34) \\
\hline \multicolumn{5}{|l|}{ 2-D } \\
\hline & $\left(\rho_{s} / \rho_{o}\right)(\varepsilon / k T)^{2 / n}$ & $\left(\rho_{F} / \rho_{o}\right)(\varepsilon / k T)^{2 / n}$ & $P / \rho_{o} k T_{m}$ & $\left(\rho_{o} \sigma^{2}=2 / \sqrt{3}\right)$ \\
\hline \multirow[t]{2}{*}{$n=\infty$} & 0.798 & 0.761 & 8.08 & Ref. (27) \\
\hline & \multicolumn{2}{|c|}{ One component plasma } & & \\
\hline 3-D & \multicolumn{2}{|c|}{$\left.\Gamma_{m}=(Z e)^{2} 4 \pi \rho / 3\right)^{1 / 3} / k T=155 \pm 10$} & $\left(\Delta \rho / \rho \approx 3.10^{-4}\right)$ & Ref. (46) \\
\hline 2-D & \multicolumn{2}{|c|}{$\Gamma_{m}=(Z e)^{2}(\pi \rho)^{1 / 2} / k T=125 \pm 15$} & $\left(\Delta \rho / \rho<410^{-4}\right)$ & Ref. (42) \\
\hline
\end{tabular}


calculations by Tsien \& Valleau (70). also suggest first-order melting of the 2-D LJ system. On the basis of their MD calculations Frenkel \& McTague (60) were unable to decide whether melting of the LJ system (at somewhat lower temperatures) was first-order or higher order. However, the low coverage $\left(\rho \sigma^{2}<0.8\right)$ work of Hanson \& McTague (71) did show a broad continuous transition that sharpened near close-packed coverage, perhaps becoming first-order there. Frenkel \& McTague noted that the melting behavior of the 2-D LJ system showed many of the characteristics predicted by Halperin \& Nelson and Young (72-74) for higher order melting in two-dimensional systems. More recent calculations by van Swol, Woodcock \& Cape (69), Abraham (75), and Toxvaerd (76) appear to be more compatible with first-order melting behavior.

In the case of the 2-D one-component plasma there is stronger evidence for higher order melting. Gann et al (42) locate the melting point of the 2-D OCP by a double tangent construction on the free energy curve derived from their $\mathrm{MC}$ results. These authors observe that their results are compatible with a first-order melting transition but that the free energy curves cross with a difference in slope of only $0.03 \%$ ! They are therefore unwilling to state an order for the phase transition. Recent MD calculations by Morf (59) have focused on the temperature dependence of the shear modulus in the 2-D OCP. Morf observes a sharp drop in the shear modulus for

$$
120<\Gamma<140, \quad\left(\Gamma=(2 e)^{2}(\pi \rho)^{1 / 2} / k T\right) .
$$

The temperature dependence of the shear modulus is in excellent agreement with the predictions of the Halperin-Nelson theory; the estimated melting point is $\Gamma=128$ [cf $125 \pm 15$ in Reference (42)]. The fact that theory and computer experiment agree so closely suggests strongly that the melting transition of the 2-D OCP is higher order. In this context it is interesting to note that the phase transition of the 3-D OCP $(46,47,68)$ is also exceedingly weak. The main reason to believe that it is first-order is Landau's theory of second-order phase transitions; if the dense Coulomb fluid were anisotropic, a higher order solid-fluid transition would also be possible in 3-D.

All the model systems discussed above exhibit simple melting behavior. Recently several papers have been published on computer simulations of model systems that mimic the more complicated melting behavior of certain real substances at high pressures, such as the melting curve maximum of cesium [e.g. see Stishov (77)]. A very simple model system to exhibit a melting curve maximum is the Gaussian core model $\left(\phi(r)=\phi_{o} e^{-r^{2}}\right)$, first analyzed by Stillinger (78). At low densities, the stable solid phase for the Gaussian core model is FCC, at higher 
densities $\left(\rho^{*}>0.179\right) \mathrm{BCC}$ is the more stable phase. At finite temperatures such a system will melt at both very high and very low densities. Molecular dynamics calculations on the Gaussian core model have been performed by Stillinger \& Weber (79). The MD calculations reveal a drastic drop in the melting temperature as the density is increased from $\rho^{*}=0.4$ to $\rho^{*}=1.0$. The phase diagram of this system is, however, not yet completely known.

Young \& Alder (80) performed MD calculations on systems of particles with a finite-step repulsive interaction $\phi(r)=\infty$ for

$$
r \leqslant \sigma, \quad \phi(r)=\varepsilon(\sigma<r \leqslant c \sigma), \quad \phi(r)=0, \quad r>c \sigma .
$$

The MD calculations revealed melting curve maxima in two-dimensional systems with $c=1.2$ and $c=1.9$. These model systems also exhibited phase transition between solids of the same symmetry but of different density. Depending on the value of $c$, these solid-solid coexistence curves ended either in a critical point or in a solid-solid-liquid triple point.

Ogura et al (38) have performed MC calculations on a quite realistic 3-D model for a system showing a melting curve maximum. In this "two-species soft-core model," it is assumed that all particles can exist in either a "ground-state" with energy $(-\varepsilon)$ or an "excited state" with energy $(+\varepsilon)$. The particles interact through the well-known $r^{-12}$ potential, but the size of the excited particles $\left(\sigma^{E}\right)$ is smaller than that of particles in the ground state $\left(\sigma^{G}\right)$. The general interaction between 2 particles is of the form

$$
\phi\left(r_{i j}\right)=\varepsilon\left(\frac{\sqrt{\sigma^{i} \sigma^{j}}}{r}\right)^{12} .
$$

Ogura et al choose $\sigma^{E}=0.8 \sigma^{G}$. The melting curve is located using the single-occupancy cell method. The phase diagram shows an FCC to FCC transition at low temperature. The solid-solid coexistence curve presumably ends in a triple point. The melting curve of the less dense solid exhibits a maximum. Neither of the models mentioned above showed any evidence of a liquid-liquid phase transition. To conclude this section on the thermodynamics of melting, we should devote a few words to the most common experimental phenomenon, i.e. the melting and freezing of molecular fluids. Surprisingly enough, very little work seems to have been done in this direction. We are unaware of any systematic studies of the melting properties of even the simplest molecular model fluids, not to mention systems forming plastic crystals [however, see Reference (78)]. It seems unlikely that this situation will persist very long. 


\section{THE KINETICS OF FREEZING AND MELTING}

The thermodynamics of the solid-fluid transition tells us little about the mechanism of this phase change. In fact, for a first-order transition, there is nothing special about the properties of either the solid or the fluid phase at coexistence (and, in a way, this is reflected in the approximate nature of many of the melting criteria discussed in the previous section). Experimentally, it is virtually impossible to follow the actual breakup of a lattice or the nucleation of solid from a homogeneous liquid, because such processes typically occur on microscopic time scales $\left[\theta\left(10^{-12} \mathrm{~s}\right]\right.$. This is, however, precisely the time domain that can be conveniently studied in computer simulations. In this section we review some of the Molecular Dynamics calculations that have been done to study melting or homogeneous nucleation. Monte Carlo simulations are of limited value for such studies because both melting and freezing involve cooperative motions; in an MC calculation, the path of a system through phase space in no way resembles the actual dynamical trajectory. That this may indeed affect the melting properties of a system is demonstrated in a paper by Briant \& Burton (81) on the solid-fluid transition in microclusters. These authors observe that, in an MD simulation, solid microclusters have to be superheated before they melt, suggesting a kinetic barrier to melting. MC calculations on the same systems were found to show no such effect.

Although melting is a very common phenomenon in MD simulations (after all, most simulations of fluids start out from an overexpanded solid configuration), relatively few studies have focused on the melting mechanism. Cotterill and co-workers have made an extensive study of the role of dislocations in 2-D and 3-D melting [for a recent review see (82) and references therein]. It was observed that, in a 2-D LJ system, dislocation pairs proliferate spontaneously at the melting point. As the (free) energy needed to form a dislocation is a function of the number of dislocations already present, dislocation-mediated melting may be expected to be a strongly cooperative phenomenon. This is indeed borne out by the MD calculations of Cotterill and co-workers. These authors have also tested some of the predictions of the dislocation-mediated melting picture $(83,84)$. In this picture, the fluid phase is considered as a "solid saturated with dislocations." Many of the equilibrium and transport properties of fluids close to the melting curve can be explained, at least to within an order of magnitude, using this picture. MD studies of dislocation-mediated melting have thus far been limited to (2-D and 3-D) Lennard-Jones systems. Some studies were done to assess the effect of system-size and periodic boundary conditions (83). In 2-D 
microcrystals with free boundaries it was found that dislocation pairs formed predominantly in the solid-like core (83), but later studies on 3-D crystal slabs (82) showed preferential generation of dislocations at the surface.

Surface melting in a 3-D LJ crystal has been studied by Broughton \& Woodcock (85). These authors observe melting of the $(1,0,0)$ surface of LJ crystals at temperatures well below the triple point-the top layer melts at

$$
T^{*} \approx 0.49 ; T_{T R I P L E}^{*}=0.68 \pm 0.02 \text {. }
$$

Premelting phenomena of a somewhat different nature have been observed by Alder et al (86). These authors observed cooperative diffusion in hard-disk and hard-sphere solids close to their melting point. In these infrequent diffusion events, a small number of nearest neighbors are shifted in a closed loop. Very close to melting the displacements appear to become more complex.

In contrast to melting, nucleation is not a phenomenon that is readily observed in computer simulations, at least in 3-D. In the early MD calculations on the hard-sphere solid-fluid transition, Alder \& Wainwright observeci occasional jumping back and forth between the solid and the fluid branch of the HS equation of state. The fluid to solid jump became exceedingly rare as the system size was increased, suggesting that the observed phenomenon is closely related to the effect of the periodic boundary conditions. The first example of solidification in a fluid with free boundaries is the study by de Wette et al (87) on the crystallization of a 2-D Lennard-Jones system. In this simulation, the temperature of a random array of $400 \mathrm{LJ}$ disks, initially at $T^{*}=0$, was allowed to evolve freely (actually, a friction term was included in the equation of motion to control the temperature). Crystallization occurred readily. Initially several crystallites formed, which later on merged to form an almost defect-free triangular lattice. Crystallization in 3-D systems is much harder to achieve. Nucleation in a 3-D LJ system was first observed accidentally by Rahman, Mandell \& McTague (88) in the course of an MD study of amorphous LJ systems. Studies of crystal nucleation were later performed by the same authors $(89,90)$ under "experimental" conditions that favor crystal nucleation. Even so, it may take many tens of thousands of time steps before a nucleation event takes place. The actual nucleation process takes place rapidly-within $6 \tau\left[\tau=\left(\mathrm{m}^{2} / \varepsilon\right)^{1 / 2}\right]$-although subsequent annealing may take a long time. Several methods of monitoring nucleation were employed by Mandell et al. It was found that the first sign of nucleation was a rapid increase of the structure factor $S(k)$ at values of $k$ corresponding to 
reciprocal lattice vectors of the incipient solid. Some $5 \tau$ later the pressure would start to drop and the temperature would start to increase. Analysis of the main Bragg peak of the resulting solid revealed that an FCC phase had nucleated in systems of 108 particles, but BCC phases had formed for $N=256$ and $N=500$ (these BCC phases would convert to $\mathrm{FCC}$ if the system temperature was quenched after the crystallization had taken place). It should be stressed that FCC and not BCC is the stable solid phase of the LJ fluid. Nucleation of a BCC phase is, however, in accordance with Ostwald's "step rule." In a recent paper Alexander \& McTague (91) have analyzed freezing in the framework of the Landau theory. These authors reach the conclusion that, close to the melting line, BCC-like fluctuations in the fluid are uniquely favored on the basis of general symmetry arguments. The Landau theory suggests that, for "weak" first-order transitions, BCC should be the stable solid phase at coexistence and, even when other solid phases are actually more stable, nucleation will occur preferentially into a metastable BCC phase. It should be added that these arguments are expected to be valid close to the coexistence curve; they need not apply for strongly supercooled fluids. In the calculations of Mandell et al nucleation typically occurred at $T^{*} \approx 0.5 T_{m}^{*}$. It was found that the "point of no return" for a nucleation event was some $1.4 \tau$ after the onset of the fluctuation in the structure factor. The critical nucleus was estimated to contain some 40-70 particles, although fairly long-range structural ordering was observed in the fluid surrounding the nucleus.

Nucleation in a soft-core fluid has been observed by Tanemura et al $(92,93)$. A somewhat different method of analysis was employed by these authors, namely the study of the nature of the Voronoi polyhedra in the nucleated phase. One small $(N=108)$, strongly supercooled system was observed to form an FCC solid. For larger $(N=500)$, less supercooled systems, only the BCC phase was found to nucleate. As in the $\mathrm{LJ}$ case, BCC is not the stable solid phase of coexistence.

Stillinger \& Weber (79) have studied the crystallization of a system of particles with "Gaussian core" interaction, i.e.

$$
\phi(r)=\phi_{0} \exp \left(-r^{2}\right) \text {. }
$$

Nucleation of a BCC phase is observed upon slow cooling at a temperature some $30 \%$ below $T_{m}$. In contrast to the cases mentioned above, BCC is the stable solid phase of the Gaussian core system at the densities studied by Stillinger $\&$ Weber.

Nucleation of liquid "rubidium" $(N=500)$ has recently been studied by Hsu \& Raman (94). The stable solid phase of $\mathrm{Rb}$ at melting is BCC and this phase was observed to nucleate with considerably greater ease 
than Lennard-Jones. The Voronoi polyhedron method was used to analyze the nucleated phase. It should be mentioned that in these calculations the fluid was rapidly supercooled to less than $0.2 T_{m}$ and thereupon allowed to evolve freely. The same method of cooling was recently used by Hsu \& Rahman in a study of the nucleation properties of fluids of 500 particles interacting through Lennard-Jones, truncated Lennard-Jones, and truncated rubidium potentials (95). In all these cases it was observed that an FCC solid was eventually formed. Hsu \& Rahman noticed that the full-potential systems tended to nucleate more easily than the truncated-potential systems. To compare directly with the results of Mandell et al, Hsu \& Rahman also studied an LJ fluid at about the same density and degree of supercooling as were used in Reference (90). In this case Hsu \& Rahman found that the nucleated solid has an imperfect FCC structure. It is not obvious at this stage whether the discrepancy between the results of Reference $(90,95)$ are due to different methods of analysis or that indeed different phases were found to nucleate.

All in all there is still alot to be learned about the different factors that influence nucleation. Clearly the intermolecular potential favors certain nucleation routes over others, but little is known about the effect of the rate of cooling or of the degree of supercooling on nucleation. Virtually nothing is known about homogeneous nucleation in molecular fluids or about inhomogeneous nucleation in any system.

\section{METASTABLE FLUIDS AND AMORPHOUS SOLIDS}

For most 3-D systems discussed in the last section, an appreciable degree of supercooling is required in order for nucleation to occur on the time scale of an MD simulation. In fact, for moderately supercooled fluids nucleation is the exception and metastability is the rule. As the temperature is decreased [or the density is increased (96)] the rate of nucleation will initially increase because the concentration of critical nuclei increases, while the size of the critical nucleus decreases. As the temperature is decreased further, the rate of nucleation will eventually decrease sharply, because at low temperatures it becomes increasingly difficult to cross the nucleation barrier. [For a discussion of the temperature dependence of the nucleation rate in a Lennard-Jones system, see (84).] A study of the properties of supercooled fluids is only meaningful if the time it takes to achieve metastable equilibrium is short compared to typical nucleation times. The extent (or even existence) of the metastable branch depends on the nature of the intermolecular forces 
and the dimensionality of the system. If nucleation is nowhere a serious competitor of structural relaxation (e.g. in the overcompressed hardsphere fluid) one may reach a point where the dynamical properties of the system appear more solid-like than fluid-like on the time scale of a typical computer simulation. This region is commonly referred to as the "glass-transition" region, although it remains to be specified what the nature of the transition or, for that matter, of the "glass phase" is.

In this section we first discuss amorphous solids from a structural point of view, based on the concept of random dense packing (RDP). Subsequently we review studies that focus on the glass transition, or, to be more precise, on the question: What features distinguish the metastable fluid phase from the amorphous solid? We want to emphasize that the nature of the glass transition is at present not well understood. Available simulation data often have been interpreted very differently by different authors. We realize that under these circumstances even the way in which we organize the presentation of the relevant material reflects a personal bias that is not necessarily shared by the authors of the papers we quote.

As we mentioned in the introduction, mechanical simulation of many-body systems predates numerical simulation. Yet the most extensive investigation of a mechanical model was undertaken at a time when machine calculations had already made their debut. Bernal and coworkers (1-7) and subsequently Scott and co-workers (8-12) studied large assemblies of randomly packed ball bearings as a model for a hard-sphere fluid. Although Bernal considered these random closepacked assemblies as models for a liquid, they probably bear a closer resemblance to the amorphous solid phase. The most remarkable aspect of the ball bearing assemblies studied by Bernal and Scott is probably the very existence of a well-defined density of random dense packing, $\rho_{R D P} / \rho_{o}=0.860$. The local geometry that corresponds to the RDP state has been analyzed by Bernal. It was found to be radically different from that of the regular close-packed solid. The average number of particles in contact with a given particle is $\sim 6.4$ (cf 12 for an FCC lattice). In the RDP structure, the tetrahedron is by far the most commonly used ( $\sim 73 \%)$ of the elementary building blocks (the five "canonical" polyhedra). It should be noted that the packing-fraction of a tetrahedron is some 5\% higher than that of a regular close-packed structure, but structures of tetrahedra are not space filling.

Alternatively, the local geometry of an amorphous solid can be characterized by means of Voronoi polyhedra. This method has been used by Finney $(13,97)$. Finney found that in random dense-packed structures, distorted icosahedra were the most prevalent polyhedra. In 
this context it is interesting to note that Alexander \& McTague (91) observed that icosahedral structures are favored by the Landau theory whenever the nucleation of a BCC solid is inhibited for some reason.

The fact that a random dense-packed state can be made in a reproducible manner (by "shaking" and "compressing" a heap of randomly poured ball bearings) suggests that this state may represent the ultimate structure that can be reached by compressing a metastable hard-sphere fluid. Although preparing an RDP structure of ball bearings is easy, analyzing it can be exceedingly tedious. For this reason several algorithms have been proposed to generate RDP structures numerically [this subject has been reviewed by Finney (97) and, more recently, by Hoare (25)]. The most straightforward (though not necessarily most economical) numerical method to generate an RDP structure is to slowly compress a hard-sphere fluid in an MD simulation. This method has been pursued by Woodcock (98). For a system of 500 hard spheres, his calculations yield an estimate of $\rho^{*} / \rho_{o}=0.8605 \pm 0.003$ for the maximum density of the amorphous branch. This value agrees within experimental error with the best estimate of the RDP density of an assembly of real spheres: $\rho^{*}{ }_{R D P}=0.8597 \pm 0.004(12,13)$.

One obvious question is whether the hard-sphere RDP structure is in any way typical for atomic glasses. The pair distribution function of the hard-sphere RDP system shows a split second peak similar (but not identical) to the ones observed experimentally in $\mathrm{Ni}-\mathrm{P}$ alloys (99). It has, however, been argued by Hoare (25) that the softness of the intermolecular potential may introduce qualitatively new features in the medium-range structure of glasses. Thus far we have been discussing dense random packing in three-dimensional hard-sphere systems. Very little is known about dense random packing in other systems. Stillinger et al (14) estimated the density of random close-packing in a hard-disk system, using a mechanical model. They obtained: $\rho_{R D P} \approx 0.89 \rho_{o}$. It is, however, not obvious whether this state is indeed well defined (unlike in 3-D, the densest local packing in 2-D is also the regular packing). We are not aware of any mechanical simulations of random dense packing for nonspherical molecules. Anyone who has ever observed a heap of needles or a bowl of potato chips will intuitively guess that, unlike the packing fraction for regular close packing, the random close-packing fraction will depend strongly on the anisotropy of the molecular shape. Is the random close-packing fraction a maximum for spheres? It seems probable, but "experimental" data are lacking.

Before discussing computer simulations of metastable fluids and amorphous solids we have to consider once more the possible effect of system size, periodic boundary conditions, and finite length of the 
simulations. Not only may the system size affect the (metastable) equilibrium properties of system under consideration, it may also change the degree of metastability. For instance, Alder \& Wainwright observed that a system of 32 hard spheres can hop back and forth between the solid and the metastable fluid branches, whereas for larger systems (e.g. $N=500$ ), transitions from the metastable fluid to the solid branch are never observed. As has already been indicated in the previous section, the smallness of a periodic system may not only influence the rate of nucleation from a metastable fluid but even favor a nucleation route that is not observed in a larger system [e.g. FCC instead of BCC for $N=108$ soft-core $(92,93)$ and $L J(89)$ systems]. It should also be noted that metastable fluids may nucleate to form misaligned, defect-rich crystals. For small systems the "equation of state" of such imperfect crystals may differ appreciably from that of the equilibrium solid and the imperfect crystal may be mistaken for a distinct phase. Alder \& Wainwright (63) analyzed the configuration of a 32-particle hard-sphere system on the "extended fluid branch" and found that it was in fact a misaligned HCP crystal.

The system size also affects the time scales for equilibration of the metastable fluid. The metastable phase can only have well-defined "equilibrium" properties if $\tau_{N U C L} \gg \tau_{E Q} \ll \tau_{R U N}$, where $\tau_{N U C L}$ and $\tau_{E Q}$ are the characteristic time scales for nucleation and equilibration, and $\tau_{R U N}$ is the duration of a simulation. As the glass transition is approached, the condition $\tau_{E Q} \ll \tau_{R U N}$ breaks down and the system starts behaving nonergodically. We will come back to this point when we discuss the role of time scales in the glass transition.

Numerous computer studies (both MC and MD) have been made of the properties of metastable fluids and amorphous solids. Actually most studies focused on either the metastable branch close to freezing or the dense amorphous solid. The region in between has been somewhat neglected (partly because the risk of nucleation is greatest in this region). The equation of state of a metastable hard-sphere fluid has been studied by Alder \& Wainwright (63) (MD on 32 particles) and Wood \& Jacobson (28) (NVT-MC on 32 particles) and Wood (41) (NPT-MC on 32 particles). MD calculations on a system of 500 hard spheres in the metastable fluid phase were performed by Woodcock $(98,100)$. There seem to be significant differences between the high density equations of state determined by Woodcock and by Alder \& Wainwright (and Wood's results are somewhat different from both the others). This perhaps explains why Gordon, Gibbs \& Fleming (101), who analyzed the Alder-Wainwright data, reach conclusions concerning the hard-sphere glass transition that are quite different from Woodcock's. Gordon et al observe that $(p / \rho \mathrm{k} T)^{-1}$ shows a break at a density 
$\rho=0.806 \rho_{o}$. The low density branch extrapolates to zero (=infinite pressure) at $\rho=0.883 \rho_{o}$, the high density branch goes to zero at $\rho=0.869 \rho_{o}$. Gordon et al interpret $\rho=0.883 \rho_{o}$ as the density of random close packing of a metastable fluid in equilibrium. The break in slope at $\rho=0.806 \rho_{o}$ is attributed to the fact that above this density the metastable fluid can no longer equilibrate during an MD run; i.e. this "transition" is considered to be purely kinetic in nature. Woodcock, however, finds no evidence for such a break in the equation of state. Rather, he suggests that there is change in slope of the excess heat-capacity $C p^{+}\left[=\left(\partial \rho^{-1} / \partial p^{-1}\right)_{T}\right.$ for hard spheres $]$ at a density $\rho=0.707 \rho_{o}$. Woodcock argues that this change of slope reflects a third-order thermodynamic transition. The argument for the occurrence of a thermodynamic transition of higher order is based on the hypothesis that the full virial equation of state has its first singularity at $\rho_{o}$ and not at $\rho_{R D P}$ [see Woodcock (102) and Baram \& Luban (103)]. If this is indeed the case, then any deviations of the "experimental" equation of state of the hard-sphere system from the full virial equation of state must be due to a (higher order) thermodynamic transition. As this is, to our knowledge, the only computer study that suggests a themodynamically well-defined glass transition, the underlying hypotheses put forward by Woodcock deserve to be studied carefully. These hypotheses are that $(a)$ the full virial equation of state does indeed diverge at $\rho_{o},(b)$ the empirical equation of state proposed by Woodcock (102) is essentially identical to the full virial equation, and $(c)$ for $\rho>0.707 \rho_{o}$ the hard-sphere system can still reach metastable equilibrium during an MD run. (This last point is not obvious, as Woodcock suggests that the fluidity $\left(\eta^{-1}\right)$ and self-diffusion constant $(D)$ of the metastable fluid vanish around $\rho \approx$ $0.707 \rho_{o}$ ). The problem with the analysis by Gordon et al (101) seems to be that it is based on results for a 32-particle system [which eventually even crystallized (63)]. Such a system is too sensitive to periodic boundary effects to be truly amorphous.

Several authors have studied the amorphous phase of continuous potential systems, in particular the 3-D Lennard-Jones system $(88,96,104-110)$, the soft-core system $(66,111)$, and the Gaussian core system (112). It should be noted that in all these systems crystal nucleation has also been observed. Consequently, if such systems are cooled or compressed too slowly, nucleation rather than vitrification will take place. In fact, in the real world the noble gas liquids (of which the $\mathbf{L J}$ fluid is supposed to be a fair model) have never been observed to vitrify. More realistic models of actual glass-forming substances, in particular molten salts and silicates, have also been studied $(108,113-$ 115). In the present section we focus on the less realistic but simpler systems because they exhibit most of the qualitative features of the 


\section{4}

computer glass transition, without the complicating features of an ionic multicomponent system.

Most MD or MC studies on the amorphous state properties of the simple potential systems mentioned above seek to give an answer to the following questions:

1. What properties (structural, transport, or thermodynamical) distinguish the vitreous state from the metastable fluid?

2. Do the properties of the vitreous state depend on the way in which it was prepared?

The metastable branch of the soft-core fluid has been studied by Hiwatari et al $(66,111)$. It was observed that the diffusion constant of the metastable soft-core fluid becomes unobservably small for densities higher than $\rho \approx 1.35(k T / \varepsilon)^{1 / 4}$ or, equivalently, $T^{*}=0.63 T_{m}^{*}$. Moreover, beyond this density Hiwatari et al found a marked system-size dependence of the equation of state [see, however, Reference (92), which discusses nucleation in these systems]. However, no evidence is found for any dependence of the properties of the amorphous state on the mode of preparation (cooling or compressing). The radial distribution function gradually develops a split second peak for $\rho>1.3(k T / \varepsilon)^{1 / 4}$ (but some high density $g(r)$ 's actually suggest that an FCC phase has formed in the $N=108$ system). Hiwatari et al initially identified $\rho^{*}=1.35$ $(k T / \varepsilon)^{1 / 4}$ as the glass transition density. However, subsequent analysis (111), revealed that the diffusion constant at high densities fits well to an equation of the form

$$
D^{*}=C \exp \left[-A /\left(V^{*}-V_{o}^{*}\right)+f\left(V^{*}\right)\right]
$$

with

$$
V_{o}^{*}=0.57\left(\rho=1.75(k T / \varepsilon)^{1 / 4}\right) \text {. }
$$

Hiwatari suggests that this latter density should be considered the "real" glass-transition density. In that case $T_{g} / T_{m} \approx 0.24$ rather than 0.63 ! This observation clearly illustrates that different criteria will yield different glass-transition points. In the following discussion we distinguish between $T_{g}$, the glass-transition temperature, at which diffusion and structural relaxation on the time scale of the experiment becomes frozen in and $T_{o}$ the extrapolated temperature at which $\eta, D^{-1}$ etc actually diverge. The former temperature may be expected to depend on system size and the duration of a simulation, the latter temperature should be relatively insensitive to these factors, as long as the system is large enough to have the essential properties of the amorphous state (whatever these may be). 
Vitrification in a Lennard-Jones system has been studied by MC $(105,106,109,110)$ and MD $(88,96,104,107)$ simulation. Raveche \& Streett $(105,106)$ performed extensive MC calculations on systems of 108 and $256 \mathrm{LJ}$ molecules. The supercooled fluids initially showed structural features typical for a cold amorphous phase [split second peak in $g(r)$ ] but after several millions of moves many systems crystallized into a distorted FCC phase. Some other systems did, however, relax to a (meta) stable amorphous structure. The properties of the amorphous phase were found to be reproducible to within 4\%. Molecular Dynamics calculations on a supercooled LJ fluid $(N=500)$ were performed by Rahman, Mandell \& McTague $(88,104)$. The amorphous state was prepared by rapidly quenching an initially liquid configuration at $\rho^{*}=$ 0.95 to a temperature $T^{*}=0.108$. Rahman et al note a clear splitting in the second peak of $g(r)$, but they observe that the appearance or disappearance of this "signature" of the cold, amorphous state does not coincide with the glass-transition temperature, i.e. the splitting disappears on heating because it gets blurred by thermal motions, not because of some underlying structural changes. In the amorphous solid studied by Rahman et al diffusion was at least four orders of magnitude smaller than in the liquid phase. The collective excitations (density fluctuations and transverse current fluctuations) were found to exhibit propagating phonon-like behavior, reminiscent of the collective excitations in "cold" $\left(T \ll \Theta_{D E B Y E}\right)$ quantum liquids. Vitrification in the amorphous $\mathrm{LJ}$ system took place at $T^{*} \approx 0.5$ (for $\rho^{*}=0.90$ ). The glasstransition temperature increased rapidly with increasing density; for $\rho^{*}=0.95$ the glass transition appeared to be spread out over a temperature range $0.8 \leqslant T^{*} \leqslant 0.9$.

Hudson \& Andersen (116) have investigated whether the vitrification of the LJ system can be understood in terms of the transition observed by Woodcock in the metastable hard-sphere fluid. Using the WeeksChandler-Andersen prescription to calculate the density of the hardsphere reference fluid (51), corresponding to an $\mathrm{LJ}$ system at vitrification $\left(T^{*}=0.5, \rho^{*}=0.90\right)$, Hudson \& Andersen obtain an estimate of $\rho^{*} / \rho_{o}=0.707$ for the density of the equivalent hard-sphere fluid. This estimate agrees well with the density at which Woodcock observed a break in the excess heat capacity of the hard-sphere fluid.

NPT-MC calculations on the glass transition in a Lennard-Jones system $(N=108)$ have been performed by Wendt \& Abraham (109) and Abraham (110). Amorphous solids were prepared by rapid cooling, compression, and cooling and compression. Wendt \& Abraham propose a structural criterion for the glass transition in terms of the ratio 


\section{6}

$R=g\left(r_{M I N}\right) / g\left(r_{M A X}\right)$, similar in spirit to the freezing criterion of Raveche, Mountain \& Streett (56). It is argued that, independent of the mode of preparation, $R \approx 0.14$ at the glass transition. The density of the hard-sphere reference system (determined using the WCA method), corresponding to a Lennard-Jones fluid with $R \approx 0.14$, is $\rho^{*} / \rho_{o} \approx 0.721$. This is in fair agreement with the results of Woodcock (98) and Hudson \& Andersen (116). In a subsequent paper (110) Abraham explores further criteria for the glass transition. He argues that, irrespective of the mode of preparation, the density and enthalpy of an $(N=108) \mathrm{LJ}$ system exhibit a change in slope at the same point where $R$ does so. If this point is interpreted as the glass transition, the density of the corresponding hard-sphere reference system agrees well with the value quoted by Woodcock. (Abraham notes, however, that, in general, $R \neq$ 0.14 at this transition.) The pair distribution function of a strongly supercooled $\mathrm{LJ}$ system $\left(T^{*}=0.1, P^{*}=1.0\right)$ is found to exhibit a small shoulder, at $r \approx 1.45 a$ [where $a$ is the position of the first maximum of $g(r)$ ]. Abraham considers the presence of this shoulder as evidence for a distorted FCC-like structure of the Lennard-Jones glass. This observation runs counter to almost every current picture of the prevalent geometries of amorphous close packing. Unfortunately, Abraham does not discuss the possibility of partial crystallization of the system. In view of the results of Raveche \& Streett $(105,106)$ and of Hsu \& Rahman (95) nucleation would seem a serious possibility, particularly for such a small $(N=108)$ system.

Support for the criterion $g\left(r_{M I N}\right) / g\left(r_{M A X}\right) \approx 0.14$ at $T_{g}$ comes from MD calculations by Stillinger \& Weber (112) on a Gaussian core model $(N=432)$. This system was slowly cooled from the fluid phase down to $T^{*}=0$ (at constant density $\rho^{*}=1.0$ ). The thermodynamic properties of the resulting $T^{*}=0$ state did not appear to depend systematically on the cooling rate. It was found that diffusion became negligible at a temperature $T_{g}^{*} \approx 0.28 T_{F}^{*}$. Particularly puzzling is the fact that a finite latent heat appears to be associated with this transition. The pair distribution seems to be quite insensitive to the transition; in particular no second peak-splitting is observed. For a soft potential like the Gaussian core this is, however, not surprising (25).

Recently the glass transition in a LJ system $(N=216$ and $N=864)$ has been studied by Clarke (96). This paper focuses on the behavior of experimetally observable quantities close to the glass transition. Several different compression and cooling methods were employed for both $N=216$ and $N=864$ systems. No significant dependence of the properties of the resulting amorphous solid on the preparation method was found (although nucleation was found to occur rather easily in the 
$N=216$ system). Amorphous state points were studied along the $P^{*} \approx 0$ isobar. A rather sharp drop in the expansivity $\alpha=(\partial \ln V) /(\partial T)$ was observed around $T^{*} \approx 0.29\left(=0.41 T_{f}\right)$. Below this temperature the amorphous phase $\alpha$ is comparable to $\alpha$ of the crystalline solid. The heat capacity $C^{*}$ also decreases sharply to crystalline values around $\rho^{*}=$ $0.98, T^{*} \approx 0.42 T_{f}$. The self-diffusion constant becomes negligible around this same temperature. For compression at constant temperature $\left(T^{*}=\right.$ $0.7)$ the diffusion constant vanishes around $\rho^{*} \approx 1.06$. By interpolation one would expect that the glass-transition density for $T^{*}=0.5$ must be between $\rho^{*}=0.98$ and $\rho^{*}=1.06$. This is in marked contrast to the findings of Rahman et al ( viz $\rho^{*}=0.90$ at $T_{g}^{*}=0.5$ ) (88). Clarke makes the interesting observation that the pressure dependence of the glass transition temperature obeys the (pseudo-Ehrenfest) relations for a second order phase transition. It is found that the temperature dependence of the diffusion constant fits well to the Fulcher law: $D$ $\exp \left(-B /\left(T-T_{o}\right)\right.$ ) with $T_{o}$ estimated to be $\sim 0.22$ (i.e. considerably lower than $T_{g}^{*}$ ). In view of the rather large discrepancy between the results of Clarke and those of Rahman et al it is hardly surprising that Clarke finds that the state of the $\mathrm{LJ}$ fluid at the glass transition does not correspond to a value of $\rho^{*} / \rho_{o} \approx 0.707$ in the hard-sphere reference system. Clarke concludes that the hard-sphere system is not a realistic reference system for amorphous close-packed phases of systems with soft repulsive interactions.

In spite of the many contradictory results that have been obtained on computer "glasses," several quite general remarks can be made. First of all it appears that glassy states can be prepared with properties that do not depend strongly on the method of preparation [although some dependence of the HS glass properties on the rate of densification has been observed by Woodcock (100)]. The system size apparently affects the rate of structural relaxation in the metastable system (96) and nucleation may be a serious problem in small systems $(N \leqslant 108)$; but otherwise no systematic system-size dependence of the thermodynamic properties of the amorphous phase have been observed. The glass transition is the point where transport on the time scale of the simulation vanishes. It seems to be associated with a fairly abrupt change in some of the higher derivatives of the thermodynamic properties of the system. Of course the definition of the glass transition depends on the time scale of the experiment and $\mathrm{MD}(\mathrm{MC})$ glass transitions may be expected to occur at temperatures much higher (densities much lower) than would be observed in hypothetical laboratory experiment on the same systems. There is no evidence for any structural change at the glass transition. This last point is important to note because it suggests 
that a clue to the nature of the glass phase may not be found by comparing the properties of the amorphous phase on both sides of the glass transition. Anderson (117) has suggested that an important clue about the nature of the glass phase is contained in the universal temperature dependence of transport properties in cold and supercooled fluids:

$$
D, \eta^{-1} \text { etc } \sim \exp \left[-B /\left(T-T_{o}\right)\right] .
$$

In Anderson's words: "The problem of the nature of the glass can be argued to be identical with the question what $T_{o}$ means." He notes that the universal form of the $T$ dependence of transport properties strongly suggests a Kosterlitz-Thouless-like theory (118) where the transition is mediated by some topological defects. The question then becomes: What is the nature of the defects? A possible candidate suggested by Anderson is a "dangling bond" in a random network. Chaudhari et al (119) have recently investigated the stability of different defect types in an amorphous $\left(T^{*}=0\right) \mathrm{LJ}$ system $(N=8000$, free boundaries). They find that edge dislocations are unstable [the same apparently holds for vacancies (120)] but screw dislocations are stable defects in the amorphous solid. It should be noted that the picture of a glass as a solid saturated with dislocations that are trapped has been advocated and analyzed in some detail by Cotterill and co-workers (84).

Let us conclude this section by pointing out some aspects of the amorphous state that, in our view, deserve to be studied numerically. First of all, a much more extensive study of the temperature and density dependence of transport properties in supercooled fluids seems essential. Second it may be necessary to search for systems that are less susceptible to nucleation (thus far, only the hard-sphere fluid appears to be safe). This may involve studying simple mixtures and possibly simple molecular fluids (but as noted before, nothing is known about nucleation in molecular fluids). Finally, there are the still inadequately understood anomalous properties of glasses at very low temperatures e.g. (121). MD calculations can make a valuable contribution to the understanding of the nature of low energy excitations in amorphous solids.

\section{ACKNOWLEDGMENTS}

This work was supported by grants from the National Science Foundation (NSF). We gratefully acknowledge numerous reprints, preprints, and helpful suggestions received from a great number of authors. 


\section{Literature Cited}

1. Bernal, J. D. 1964. Proc. R. Soc. London Ser. A 280:99

2. Rice, O. K. 1944. J. Chem. Phys. 12:1

3. Westman, A. E. R., Hugill, H. R. 1930. J. Am. Ceram. Soc. 13:767

4. Bernal, J. D. 1959. Nature 183:141

5. Bernal, J. D. 1960. Nature 185:68

6. Bernal, J. D., Mason, J. 1960. Nature 188:910

7. Bernal, J. D., King, S. V. 1968. In Physics of Simple Liquids, ed. H. N. V. Temperley, J. S. Rowlinson, G. S. Rushbrooke, p. 233. Amsterdam: North-Holland

8. Scott, G. D. 1960. Nature 188:908

9. Scott, G. D. 1962. Nature 194:956

10. Scott, G. D., Mader, D. L. 1964. Nature 201:382

11. Scott, G. D., Charlesworth, A. M. Mak, M. K. 1964. J. Chem. Phys. 40:611

12. Scott, G. D., Kilgour, D. M. 1969. J. Phys, D 2:863

13. Finney, J. L. 1970. Proc. R. Soc. London Ser. A 319:479

14. Stillinger, F. H., DiMarrio, E. A., Kornegay, R. L. 1964. J. Chem. Phys. 40:1564

15. Metropolis, N., Rosenbluth, A. W. Rosenbluth, M. N. Teller, A. H. Teller, E. 1953. J. Chem. Phys. 21:1087

16. Alder, B. J., Wainwright T. E. 1957. J. Chem. Phys. 27:1208

17. Wood, W. W. 1968. See Ref. 7, p. 117

18. Valleau, J. P. Whittington, S. G. 1977. In Statistical Mechanics, Part A: Equilibrium Techniques, ed. B. J. Berne, p. 137. New York: Plenum

19. Valleau, J. P. Torrie, G. M. 1977. See Ref. 18, p. 169

20. Alder, B. J., Wainwright, T. E. 1959. J. Chem. Phys. 31:549

21. Erpenbeck, J. J., Wood, W. W. 1977. In Statistical Mechanics, Part B: Time Dependent Processes, ed. B. J. Berne, p. 1. New York: Plenum

22. Kushick, J., Berne, B. J. 1977. See Ref. 21, p. 41

23. Hansen, J. P., McDonald, I. R. 1976. Theory of Simple Liquids. London: Academic

24a. Mandell, M. J. 1976. J. Stat. Phys. 15:299

24b. Hansen, J. P., Levesque, D., Weis, J.
J. 1979. Phys. Rev. Lett. 43:979

25. Hoare, M. R. 1978. J. Non-Cryst. Solids 31:157

26. Rosenbluth, M. N. Rosenbluth, A. W. 1954. J. Chem. Phys. 22:881

27. Hoover, W. G., Ree, F. H. 1968. J. Chem. Phys. 49:3609

28. Wood, W. W., Jacobson, J. D. 1957. J. Chem. Phys. 27:1207

29. Alder, B. J., Wainwright, T. E. 1962. Phys. Rev. 127:359

30. Cape, J. N., Woodcock, L. V. 1978. Chem. Phys. Lett. 51:271

31. Ladd, A. J. C. Woodcock, L. V. 1977. Chem. Phys. Lett. 51:155

32. Ladd, A. J. C. Woodcock, L. V. 1978. Mol. Phys. 36:611

33. Mayer, J. E., Wood, W. W. 1965. J. Chem. Phys. 42:4268

34. Hoover, W. G., Gray, S. C., Johnson, K. W. 1971. J. Chem. Phys. 55:1128

35. Hoover, W. G., Ree, F. H. 1967. $J$. Chem. Phys. 47:4873

36. Toxvaerd, S. 1978. J. Chem. Phys. 69:4750

37. Hoover, W. G., Ross, M., Johnson, K. W., Henderson, D., Barker, J. A., Brown, B. C. 1970 . J. Chem. Phys. 52:4931.

38. Ogura, H., Matsuda, H., Ogawa, T., Ogita, N., Ueda, A. 1977. Prog. Theor. Phys. 58:419

39. Landau, L. D., Lifshits, E. M. 1958. Statistical Physics, London: Pergamon

40. Hoover, W. G., Alder, B. J. 1967. J. Chem. Phys. 46:686

41. Wood, W. W, 1970. J. Chem. Phys. 52:729

42. Gann, R. C., Chakravarty, S., Chester, G. V. 1979. Phys. Rev. B 20:326

43. Lindemann, F. A. 1910. Z. Phys. 11:609

44. Ross, M., Alder, B. J. 1966. Phys. Rev. Lett. 16:1077

45. Young, D. A., Alder, B. J. 1974. $J$. Chem. Phys. 60:1254

46. Pollock, E. L., Hansen, J. P. 1973. Phys. Rev. A 8:3110

47. Ross, M. 1969. Phys. Rev. 184:233

48. Hansen, J. P., Verlet, L. 1969. Phys. Rev. 184:151

49. Hansen, J. P., Schiff, D. 1973. Mol Phys. 25:1281

50. Barker, J. A., Henderson, D. 1967. J. Chem. Phys. 47:4714 
51. Weeks, J. D., Chandler, D., Andersen, H. C. 1971. J. Chem. Phys. 54:5237

52. Longuet-Higgins, H. C., Widom, B. 1964. Mol. Phys. 8:549

53. Verlet, L. 1968. Phys. Rev. 165:201

54. Hockney, R. W., Brown, T. R. 1975. J. Chem. C. 8:1813

55. Henderson, D. 1977. Mol. Phys. $34: 301$

56. Raveche, H. J., Mountain, R. D., Streett, W. B. 1974. J. Chem. Phys. 61:1970

57. Streett, W. B., Raveche, H. J., Mountain, R. D. 1974. J. Chem. Phys. 61:1960

58. Hansen, J. P., Pollock, E. L. 1975. J. Chem. Phys. 62:4581

59. Morf, R. H. 1979. Phys. Rev. Lett. 43:931

60. Frenkel, D., McTague, J. P. 1979. Phys. Rev. Lett. 42:1632

61. Hoover, W. G., Young, D. A., Grover, R. 1972. J. Chem. Phys. 56:2207

62. Wainwright, T., Alder, B. J. 1958. Nuovo Cimento 9: Suppl. 1, p. 116

63. Alder, B. J., Wainwright, T. E. 1960. J. Chem. Phys. 33:1439

64. Wood, W. W., Parker, F. R., Jacobson, J. D. 1958. Nuovo Cimento 9: Suppl. 1, p. 133

65. Hansen, J. P. 1970. Phys. Rev. A 2:221

66. Hiwatari, Y., Matsuda, H., Ogawa, T., Ogita, N., Ueda, A. 1974. Prog. Theor. Phys. 52:1105

67. Brush, S. G., Sahlin, H. L., Teller, E. 1966. J. Chem. Phys. 45:2102

68. Hansen, J. P. 1973. Phys. Rev. A 8:3096

69. van Swol, F., Woodcock, L. V., Cape, J. N. 1980. J. Chem. Phys. In press

70. Tsien, F., Valleau, J. P. 1974. Mol. Phys. 24:177

71. Hanson, F. E., McTague, J. P. 1980. J. Chem. Phys. In press

72. Halperin, B. I., Nelson, D. R. 1978. Phys. Rev. Lett. 41:121

73. Nelson, D. R., Halperin, B. I. 1979. Phys. Rev. B 19:2457

74. Young, A. P. 1979. Phys. Rev. B 19:1855

75. Abraham, F. F. 1980. Phys. Rev. Lett. 44:463

76. Toxvaerd, S. 1980. Phys. Rev. Lett. 44:1002

77. Stishov, S. M. 1975. Sov. Phys. Usp. 17:625
78. Stillinger, F. H. 1976. J. Chem. Phys. 65:3968

79. Stillinger, F. H., Weber, T. A. 1978. J. Chem. Phys, 68:3837

80. Young, D. A., Alder, B. J. 1979. J. Chem. Phys. 70:473

81. Briant, C. L., Burton, J. J. 1975. J. Chem. Phys. 63:2045

82. Cotterill, R. M. J. 1979. In Ordering in Strongly Fluctuating Condensed Matter Systems, ed. T. Riste. New York: Plenum

83. Cotterill, R. M. J. 1974. In Anharmonic Lattices, Structural Transitions, and Melting, ed.T. Riste. London: Noordhoff

84. Cotterill, R. M. J., Jensen, E. J., Kristensen, W. D. Paetsch, R., Esbjorn, P. O. 1975. J. Phys. Paris Colloq. 36:2-35

85. Broughton, J. Q., Woodcock, L. V. 1978. J. Phys. C 11:2743

86. Alder, B. J., Gardner, W. R., Hoffer, J. K. Philips, N. E., Young, D. A. 1968. Phys. Rev. Lett. 21:732

87. de Wette, F. W., Allen, R. E., Hughes, D. S., Rahman, A. 1969. Phys. Lett. A 29:548

88. Rahman, A., Mandell, M. J., McTague, J. P. 1976. J. Chem. Phys. 64:1564

89. Mandell, M. J., McTague, J. P., Rahman, A. 1976. J. Chem. Phys. 64:3699

90. Mandell, M. J., McTague, J. P., Rahman, A. 1977. J. Chem. Phys. 66:3070

91. Alexander, S., McTague, J. 1978. Phys. Rev. Lett. 41:702

92. Tanemura, M., Hiwatari, Y., Matsuda, H., Ogawa, T., Ogita, N., Ueda, A. 1977. Prog. Theor. Phys. 58:1079

93. Tanemura, M., Hiwatari, Y., Matsuda, H., Ogawa, T., Ogita, N., Ueda, A. 1978. Prog. Theor. Phys. $59: 323$

94. Hsu, C. S., Rahman, A. 1979. J. Chem. Phys. 70:5234

95. Hsu, C. S., Rahman, A. 1979. J. Chem. Phys. 71:4974

96. Clark, J. H. R. 1979. J. Chem. Soc. Faraday Trans. II 75:1371

97. Finney, J. L. 1975. J. Physique Paris Colloq. 36:2-1

98. Woodcock, L. V. 1976. J. Chem. Soc. Faraday Trans. II 72:1667

99. Cargill, G. S. 1970. J. Appl. Phys. $41: 12$

100. Woodcock, L. V. 1978. J. Chem. Soc. 
Faraday Trans. II 74:11

101. Gordon, J. M., Gibbs, J. H., Fleming, P. D. 1976. J. Chem. Phys. 65:2771

102. Woodcock, L. V. 1976. J. Chem. Soc. Faraday Trans. II 72:731

103. Baram, A., Luban, M. J. Phys. C

104. McTague, J. P., Mandell, M. J., Rahman, A. 1978. J. Chem. Phys. 68:1876

105. Raveche, H. J., Streett, W. B. 1976. J. Res. Natl. Bur. Stand. Sect. A 80:59

106. Raveche, H. J. 1976. Ann. NY Acad. Sci. 279:36

107. Kristensen, W. D. 1976. J. Non-Cryst. Solids 21:303

108. Angell, C. A., Cheeseman, P. A., Clarke, J. H. R., Woodcock, L. V. In The Structure of Non-Crystalline Materials, p. 191

109. Wendt, H. R., Abraham, F. F. 1978. Phys. Rev. Lett. 41:1244

110. Abraham, F. F. 1980. J. Chem. Phys. 72:359

111. Hiwatari, Y. 1978. Prog. Theor. Phys.
$59: 1401$

112. Stillinger, F. H., Weber, T. A. 1979. J. Chem. Phys. 70:4879

113. Woodcock, L. V. 1972. Proc. Conf. Comput. Simulation, Sess. 4, Phys. Sci. San Diego, Calif., 1:847

114. Woodcock, L. V., Angell, C. A., Cheeseman, P. 1976. J. Chem. Phys. 65:1565

115. Soules, T. F. 1979. J. Chem. Phys. 71:4570

116. Hudson, S., Andersen, H. C. 1978. $J$. Chem. Phys. 69:2323

117. Andersen, P. W. In Les Houches Lectures Amsterdam: North Holland. In press

118. Kosterlitz, J. M., Thouless, D. J. 1973. J. Phys. C 6:1181

119. Chaudhari, P., Levi, A., Steinhardt, P. 1979. Phys. Rev. Lett. 43:1517

120. Bernet, C. H., Chaudhari, P., Moruzzi, V., Steinhardt, P. Submitted for publication.

121. Stevens, R. B. 1976. Phys. Rev. B $13: 852$ 